\title{
Managing rational routes to randomness
}

\section{Noemi Schmitt and Frank Westerhoff}

Working Paper No. 96

January 2015

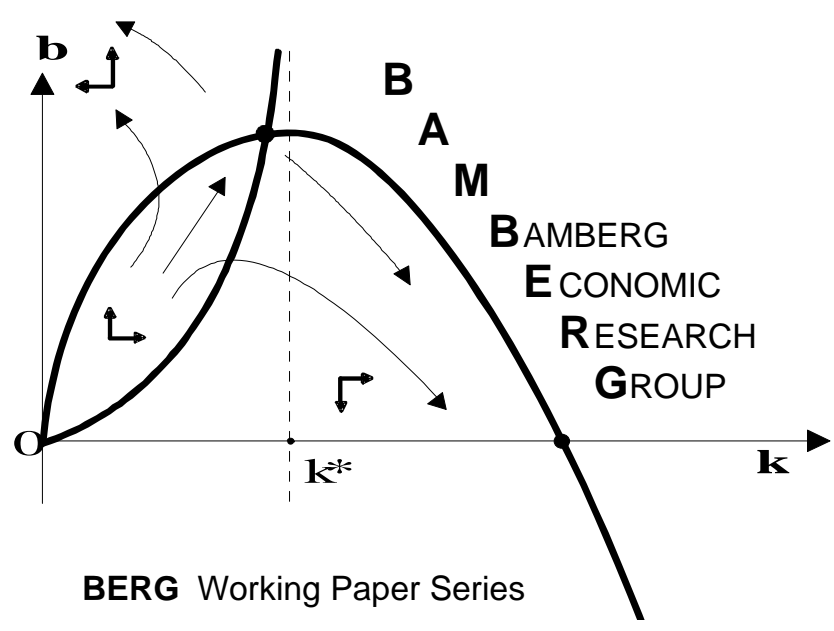

Bamberg Economic Research Group

Bamberg University

Feldkirchenstraße 21

D-96052 Bamberg

Telefax: (0951) 8635547

Telephone: (0951) 8632687

felix.stuebben@uni-bamberg.de

http://www.uni-bamberg.de/vwl/forschung/berg/

ISBN 978-3-943153-13-2 


\title{
Managing rational routes to randomness*
}

\author{
Noemi Schmitt and Frank Westerhoff ${ }^{* *}$ \\ University of Bamberg, Department of Economics, \\ Feldkirchenstrasse 21, 96045 Bamberg, Germany
}

\begin{abstract}
Within the seminal cobweb model of Brock and Hommes, firms adapt their price expectations by a profit-based switching behavior between free naïve expectations and costly rational expectations. Brock and Hommes demonstrate that fixed-point dynamics may turn into increasingly complex dynamics as the firms' intensity of choice increases. We show that policy-makers are able to manage rational routes to randomness by adjusting profit taxes. As suggested by our analytical and numerical analysis, policy-makers should increase (decrease) profit taxes if destabilizing expectations generate higher (lower) profits than stabilizing expectations to alter the composition of applied expectation rules and thereby to promote market stability. Our results are not restricted to cobweb models: a huge body of literature demonstrates that rational routes to randomness may emerge in many different markets.
\end{abstract}

\section{Keywords}

Cobweb models; discrete choice approach; intensity of choice; profit taxes; stability analysis; policy implications.

\section{JEL Classification}

D84; E30; Q11.

\footnotetext{
* Presented at "The $2^{\text {nd }}$ GENED Meeting”, Darmstadt, September 2014, at “The $8^{\text {th }}$ Workshop on Dynamic Models in Economics and Finance", Urbino, September 2014, and at "The $20^{\text {th }}$ International Conference on Computing in Economics and Finance”, Oslo, June 2014. We thank the participants for their stimulating feedback, in particular Roberto Dieci, Tony He, Cars Hommes, Irina Sushko and Jan Tuinstra. Research for this paper was supported by ISCH COST Action IS1104: "The EU in the new complex geography of economic systems: models, tools and policy evaluation”.
}

** Corresponding author’s email address: frank.westerhoff@uni-bamberg.de 


\section{Introduction}

International stock and foreign exchange markets are highly volatile and regularly produce severe bubbles and crashes. In this respect, the dot-com bubble and the stark up-and-down movements of the euro-dollar exchange rate are just two notorious examples of many. Real estate and commodity markets may also be subject to dramatic fluctuations. Between 2000 and 2005, for instance, housing prices in the United States almost doubled. Similarly, the prices of oil, gold and various agricultural goods occasionally display alarming boom and bust dynamics. Sometimes even the real economy undergoes major changes, after which we observe pronounced variations of central macroeconomic variables such as inflation and national income. While we have repeatedly encountered difficult economic periods in the past - detailed historical accounts can be found in Kindleberger (2000), Shiller (2005) and Reinhart and Rogoff (2009) - the recent global financial and economic crisis urgently reminds us just how vulnerable modern economies can be.

Models with heterogeneous interacting agents undoubtedly help us to gain a better understanding of the intricate behavior of many different markets. The key characteristics of these models are that agents are boundedly rational, display a rule-governed behavior and interact with each other. General surveys of this line of research are provided by Chiarella et al. (2009), Hommes and Wagener (2009) and Lux (2009). A seminal contribution in this area is the cobweb model by Brock and Hommes (1997). Note that cobweb models are important benchmark models in economic dynamics; they describe price dynamics in a competitive market for a non-storable good that takes one time period to produce. Due to the production lag, firms must form price expectations one time period ahead. An important feature of the cobweb model by Brock and Hommes (1997) is that firms endogenously switch between different expectation rules. In particular, firms can either use a free naïve or a costly rational expectation rule and update their (boundedly) rational choices according to the relative past performance of the respective rules. 
A central insight generated by this model is that an increase in the firms' intensity of choice may turn fixed-point dynamics into increasingly complex and volatile dynamics. This bifurcation structure, which Brock and Hommes (1997) call a rational route to randomness, may be understood as follows. Suppose that the demand and supply conditions are such that the model's unique steady state is stable if half of the firms use naïve expectations while it is unstable if all firms rely on naïve expectations. Moreover, note that prediction errors of naïve expectations are rather low as the price approaches its steady-state value. Close to the steady state, naïve expectations generate higher profits than costly rational expectations since they are free. Now, the intensity of choice indicates how sensitively firms react to profit differences between their expectation rules. Let us contrast two extreme scenarios. First, if the intensity of choice is low, firms react only weakly to profit differences of their expectation rules. The use of (destabilizing) naïve and (stabilizing) rational expectations is then roughly balanced, and the steady state is stable. Second, if the intensity of choice is high, naïve expectations will be more popular, and the steady state will be unstable. However, this doesn't necessarily imply that the dynamics explodes. Far from the steady state, naïve expectations are less precise and, despite being costly, firms eventually prefer rational expectations. As the price reverts to its steady state, prediction errors of naïve expectations decline. Firms return to naïve expectations, and the price starts to deviate from its steady state again. Due to the nonlinear interplay between a centrifugal force close to the steady state and a centripetal force far from the steady state, these price patterns may repeat themselves in an intricate manner.

Rational routes to randomness are a surprisingly robust bifurcation path: Goeree and Hommes (2000) generalize the Brock and Hommes (1997) model to the case of nonlinear demand and supply; in Lasselle et al. (2005) firms switch between rational and adaptive expectations; and Branch and McGough (2008) update the market shares of firms using naïve and rational expectations on the basis of replicator dynamics. Rational routes to randomness can also be observed in other model contexts. In the duopoly model by Droste et al. (2002), 
complex dynamics occur if the evolutionary competition between firms' rules is high. In another influential paper, Brock and Hommes (1998) detect rational routes to randomness in an asset-pricing model in which market participants have the choice between technical and fundamental predictors. Quite similar asset pricing dynamics is obtained in the models by Diks and van der Weide (2003) and Brock et al. (2005) in which speculators select between a large number of technical and fundamental predictors; in the models by Hommes et al. (2005) and Diks and van der Weide (2005) in which heterogeneous speculators asynchronously update their beliefs; in the model by Anuvrief and Panchenko (2009) in which different market designs ranging from market clearing setups to market maker scenarios are explored; and in the model by Chiarella et al. (2013) in which heterogeneous speculators can invest in multiple risky assets. Moreover, de Grauwe and Grimaldi (2006) discover complex endogenous dynamics in a foreign exchange market model with heterogeneous speculators. Dieci and Westerhoff (2014) notice boom and bust dynamics in a housing market model resulting from interactions between investors who follow heterogeneous rules to predict housing prices. Finally, we conclude this incomplete list of works by mentioning that Branch and McGough (2010), de Grauwe (2011), Lines and Westerhoff (2010) and Anufriev et al. (2013a) develop macroeconomic models in which agents’ switching between heterogeneous inflation and/or income expectations may lead to rational routes to randomness.

In recent years, models with heterogeneous interacting agents have received considerable empirical support. For instance, laboratory experiments, reviewed in Hommes (2011), reveal that human subjects rely on heterogeneous forecasting rules. Anufriev and Hommes (2012) manage to explain such experimental studies by models in which agents switch between different forecasting strategies. Evidence of heterogeneous expectations in agricultural markets is provided by Baak (1999) and Chavas (2000). The questionnaire evidence surveyed by Menkhoff and Taylor (2007) reveals that heterogeneous expectations are also a widespread phenomenon in financial markets. Moreover, Boswijk et al. (2007) and 
Franke and Westerhoff (2012) conclude on the basis of estimated financial market models that stock market traders switch between different expectation rules. Similar evidence is provided by Dick and Menkhoff (2013) and Goldbaum and Zwinkels (2013) for the foreign exchange market; by ter Ellen and Zwinkels (2010) for the oil market; and by Kouwenberg and Zwinkels (2014) for the U.S. housing market. Branch (2004) shows that survey data on inflation expectations can be explained by a heterogeneous expectations and switching model.

Since models with heterogeneous interacting agents are quite powerful and supported by empirical evidence, they are increasingly used as tools for conducting economic policy experiments. For instance, Westerhoff and Dieci (2006) explore the consequences of transaction taxes; Brock et al. (2009) show that additional hedging instruments may destabilize financial markets; Yeh and Yang (2010) discuss the effects of price limits; Anufriev and Tuinstra (2013) model short-selling constraints; Tuinstra et al. (2014) address the optimal size of trade barriers; and Branch and McGough (2010), de Grauwe (2011), Lines and Westerhoff (2010) and Anufriev et al. (2013) explore whether monetary policy rules may stabilize fluctuations in economic activity. What all of these papers have in common is that they study how certain policy measures influence market participants' actions, in particular their expectation formation behavior. For a survey of this research approach, see Westerhoff and Franke (2014).

The goal of our paper is to explore whether policy-makers can manage rational routes to randomness by adjusting profit taxes. Our reference point is the cobweb model by Brock and Hommes (1997). Since firms' (boundedly) rational choices of their expectation rules depend on the profits realized in the past by these rules, policy-makers can principally alter the relative fitness of expectation rules by imposing profit taxes. Profit taxes may thus allow policy-makers to change the mix of expectation rules applied and thereby to stabilize the market's dynamics. But how should policy-makers set profit tax rates? To address this problem, we extend the model by Brock and Hommes (1997) along three dimensions. First of 
all, we allow policy-makers to raise profit taxes. For simplicity, we focus on the case in which policy-makers impose a proportional tax on positive profits. Second, we work with a slightly more general cost function than that in Brock and Hommes (1997) by additionally considering fixed costs. While fixed costs are irrelevant in their model, they may have nontrivial effects on the global dynamics of our model. Finally, we account for the fact that producers may have a behavioral bias towards using simple expectation rules. According to this assumption, the fitness of naïve and rational expectation rules does not depend solely on past profits, but may also include untaxable behavioral components. All other model parts are specified as in the model by Brock and Hommes (1997).

Our main results may be sketched as follows. Rational routes to randomness require that the destabilizing naïve expectation rule is fitter than the stabilizing rational expectation rule close to the steady state. The more strongly firms perceive the fitness differentials of expectation rules, the more quickly they will switch between expectation rules, and the more complex the dynamics becomes. We show that, under certain assumptions, policy-makers have the opportunity to completely revert rational routes to randomness. The explanation for this remarkable result is as follows. If firms always make profits and if the fitness of expectation rules depends only on past profits, policy-makers are able to level fitness differences between expectation rules by taxing firms’ profits. An increase in the profit tax rate reduces fitness differences between expectation rules and thereby counteracts an increase in the intensity of choice. In general, however, the effectiveness of profit taxes depends on a number of factors. For example, firms do not always make profits, and behavioral preferences for certain expectation rules cannot be affected by profit taxes. Overall, our analytical and numerical results suggest that policy-makers should increase (decrease) profit taxes if destabilizing expectation rules produce higher (lower) profits than stabilizing expectation rules. As we will see, our analysis also reveals a number of more subtle insights. For instance, profit taxes may influence the basin of attraction of the model's steady state and create or 
destroy coexisting high-amplitude attractors.

Our policy implications are based on the cobweb model by Brock and Hommes (1997). Nevertheless, we believe that our results are of a more general nature since many economic systems are expectations feedback systems. As discussed in detail in Hommes (2013), the dynamics of many markets crucially depends on market participants' expectations which, in turn, depend on the current and past outcomes of these markets. According to the aforementioned empirical evidence, human subjects rely in many different situations on heterogeneous rules to predict future economic variables. As a result, the dynamics of the underlying economic system depends on the mix of the rules applied. If market participants change their rules with respect to past performance criteria - which is also suggested by empirical studies - then policy-makers have the opportunity to affect the relative fitness of the expectation rules applied by taxing market participants' profits, income or wealth such that stabilizing expectation rules become more popular and, consequently, the dynamics less unstable.

The remainder of this paper is organized as follows. In section 2, we generalize the cobweb model by Brock and Hommes (1997). In section 3, we present our analytical results. In particular, we derive the model's steady state and discuss how its local asymptotic stability is affected by the intensity of choice and profit taxes. In section 4, we present various numerical results to illustrate how the model's global dynamics depends on the intensity of choice and on profit taxes. In section 5, we summarize our main results and highlight a few avenues for future research.

\section{An evolutionary cobweb model with profit taxes}

In this section, we generalize the seminal cobweb model by Brock and Hommes (1997). The setup of our model is presented in section 2.1; its dynamical system is then derived in section 2.2. 


\subsection{The model's setup}

Cobweb models describe the price dynamics of a competitive market for a non-storable good that takes one time period to produce. Producers must thus form their price expectations one time period ahead. Brock and Hommes (1997) assume that firms switch between a free naïve and a costly rational expectation rule. Moreover, a firm's choice of an expectation rule depends on the relative past performance of the respective rule. For high values of the intensity of choice, the firms' rule selection behavior may cause complex endogenous dynamics, and prices may deviate substantially from their steady state. To explore whether policy-makers are able to manage such dynamics, we extend the model by Brock and Hommes (1997) along three dimensions. Besides adding profit taxes to the model, we also consider the fact that firms may face fixed costs and have a behavioral preference towards naïve expectations.

Let us turn to the details of the model. Market clearing occurs in every period. Accordingly, we have

$D_{t}=S_{t}$

where $D_{t}$ and $S_{t}$ stand for demand and supply at time step $t$, respectively. Consumer demand is assumed to be linearly decreasing in the current price $p_{t}$ and is formalized as

$D_{t}=a-b p_{t}$

where $a, b>0$. Firms need to form price expectations one period ahead and choose between two different expectation rules to determine their production decisions. Normalizing the number of firms to one, their total supply can be represented as

$S_{t}=n_{t-1}^{N} q_{t}^{N}+n_{t-1}^{R} q_{t}^{R}$

where $q_{t}^{N}$ and $q_{t}^{R}$ indicate the quantities supplied by firms holding naïve and rational expectations; $n_{t-1}^{N}$ and $n_{t-1}^{R}$ denote their market shares. 
In contrast to Brock and Hommes (1997), firms face a quadratic cost function that may also include fixed costs, i.e. $C_{t}=\frac{1}{2 c} q_{t}^{2}+d$, where $c>0$ and $d \geq 0$. Moreover, firms may have to pay profit taxes, where $0 \leq \tau \leq 1$ denotes the tax rate levied on (positive) profits. Despite our model changes, the firms' optimal supply, derived from expected profit maximization, i.e.

$\arg \max _{q_{t}} \pi_{t}^{e}=\arg \max _{q_{t}}\left\{\begin{array}{l}(1-\tau)\left(p_{t}^{e} q_{t}-C_{t}\right) \\ p_{t}^{e} q_{t}-C_{t}\end{array}\right.$

remains as in Brock and Hommes (1997) and is given by $q_{t}=c p_{t}^{e}$. In forming their price expectations, firms can either use a naïve predictor by simply taking the last observed price as a forecast, i.e. $p_{t}^{e}=p_{t-1}$, or they can use a rational expectations forecast (perfect foresight), i.e. $p_{t}^{e}=p_{t}$. Quantities supplied by firms using naïve or rational expectation rules are therefore given by

$q_{t}^{N}=c p_{t-1}$

and

$q_{t}^{R}=c p_{t}$

Naïve expectations are freely available; by contrast, rational expectations may incur positive per period information costs $F \geq 0$. In order to generate accurate forecasts, firms must have perfect knowledge about all producers' prices and beliefs. Information costs can be seen as an extra effort that firms may encounter in acquiring this knowledge. It goes without saying that information costs have no impact on the firms' optimal supply decision and have, for notational simplicity, been omitted in the firms' maximization problem (4).

The market shares of firms holding either naïve or rational expectations are updated over time according to an evolutionary fitness measure. Firms are boundedly rational in the 
sense that they tend to choose the forecasting strategy with the highest fitness which, in turn, depends on profits realized in the past. Since we also consider the firms' behavioral preference towards certain prediction strategies (e.g. Branch 2004, Franke and Westerhoff 2012, Anufriev et al. 2013b), the fitness of naïve and rational expectations is defined as

$A_{t}^{N}=\pi_{t}^{N}+B$

and

$A_{t}^{R}=\pi_{t}^{R}$

where $\pi_{t}^{N}$ and $\pi_{t}^{R}$ describe the profits realized by firms that follow naïve and rational expectations, respectively, and parameter $B \geq 0$ represents a behavioral bias towards naïve expectations. $^{1}$

If pre-tax profits are positive, profits realized by naïve producers are given by $\pi_{t}^{N}=(1-\tau)\left(p_{t} q_{t}^{N}-\frac{\left(q_{t}^{N}\right)^{2}}{2 c}-d\right)$, while profits realized by rational firms amount to $\pi_{t}^{R}=(1-\tau)\left(p_{t} q_{t}^{R}-\frac{\left(q_{t}^{R}\right)^{2}}{2 c}-d-F\right)$. Of course, firms are not required to pay profit taxes if their profits are not positive. For the two forecasting strategies, profits realized in period $t$ can be expressed by the two parted functions

$$
\pi_{t}^{N}= \begin{cases}(1-\tau)\left(\frac{1}{2} c p_{t-1}\left(2 p_{t}-p_{t-1}\right)-d\right) & \text { if } \quad \frac{1}{2} c p_{t-1}\left(2 p_{t}-p_{t-1}\right)-d>0 \\ \frac{1}{2} c p_{t-1}\left(2 p_{t}-p_{t-1}\right)-d & \text { if } \quad \frac{1}{2} c p_{t-1}\left(2 p_{t}-p_{t-1}\right)-d \leq 0\end{cases}
$$

and

\footnotetext{
${ }^{1}$ It is easy to extend our analysis to $B<0$, i.e. to the case in which firms have a behavioral preference towards rational expectations. A number of results for this scenario are sketched in footnote 2 .
} 
$\pi_{t}^{R}=\left\{\begin{array}{ll}(1-\tau)\left(\frac{1}{2} c p_{t}^{2}-d-F\right) & \text { if } \quad \frac{1}{2} c p_{t}^{2}-d-F>0 \\ \frac{1}{2} c p_{t}^{2}-d-F & \text { if } \quad \frac{1}{2} c p_{t}^{2}-d-F \leq 0\end{array}\right.$,

respectively.

Brock and Hommes (1997) determine the market shares of producers that choose naïve or rational expectations via the discrete choice approach taken by Manski and McFadden (1981). Therefore, we have

$$
n_{t}^{N}=\frac{\exp \left(\beta A_{t}^{N}\right)}{\exp \left(\beta A_{t}^{N}\right)+\exp \left(\beta A_{t}^{R}\right)}
$$

and

$$
n_{t}^{R}=\frac{\exp \left(\beta A_{t}^{R}\right)}{\exp \left(\beta A_{t}^{N}\right)+\exp \left(\beta A_{t}^{R}\right)} .
$$

Note that the greater the fitness of an expectation rule, the more firms will rely on it. Parameter $\beta \geq 0$ may be regarded as the firms' intensity of choice. It measures how sensitive firms are to selecting the most attractive predictor. For $\beta=0$, firms do not observe any fitness differentials between the two forecasting strategies, and both market shares will equal $1 / 2$. The higher the intensity of choice, the more firms will select the prediction strategy with the greatest fitness. For $\beta=\infty$, fitness differentials are observed perfectly and all firms will choose the predictor that yields the greater fitness.

\subsection{The model's dynamical system}

Let us next derive the model's dynamical system. Combining (1)-(3) with (5)-(6) yields

$$
a-b p_{t}=n_{t-1}^{N} c p_{t-1}+n_{t-1}^{R} c p_{t}
$$

Since $n_{t-1}^{N}+n_{t-1}^{R}=1$, the model's steady state price results as 


$$
p^{*}=\frac{a}{b+c}
$$

Moreover, solving (13) explicitly for $p_{t}$ reveals that

$$
p_{t}=\frac{a-n_{t-1}^{N} c p_{t-1}}{b+c n_{t-1}^{R}} \text {. }
$$

Before we continue, let us briefly comment on the model's time structure. The current price $p_{t}$ depends on past market shares $n_{t-1}^{N}$ and $n_{t-1}^{R}$. Once price $p_{t}$ has been fixed, profits generated by the two expectation rules can be identified and, as a result, the new market shares $n_{t}^{N}$ and $n_{t}^{R}$ follow from (11) and (12). The next period's price $p_{t+1}$ is determined with the new market shares and so on. In this sense, prices and market shares coevolve over time.

It is convenient to rewrite the model's dynamical system in deviations from the steady state price $p^{*}$, i.e. to introduce $\tilde{p}_{t}=p_{t}-p^{*}$. Moreover, we define the difference between the market shares of the two expectation rules as $m_{t}=n_{t}^{R}-n_{t}^{N}$, where $m_{t}=1 \quad\left(m_{t}=-1\right)$ corresponds to the case in which all producers hold rational (naïve) expectations. As in the original model by Brock and Hommes (1997), the dynamics of our model is driven by a twodimensional nonlinear map. If we denote by $A_{t}=A_{t}^{R}-A_{t}^{N}$ the relative fitness of rational expectations versus naïve expectations, we obtain for our setup the dynamical system

$$
\tilde{p}_{t}=\frac{-\left(1-m_{t-1}\right) c \tilde{p}_{t-1}}{\left(m_{t-1}+1\right) c+2 b}
$$

and

$$
m_{t}=\tanh \left(\frac{1}{2} \beta A_{t}\right)
$$

where 


$$
A_{t}= \begin{cases}(1-\tau)\left(\frac{c}{2}\left(\tilde{p}_{t}-\tilde{p}_{t-1}\right)^{2}-F\right)-B & \text { if } \mu_{t}>0 \wedge \omega_{t}>0 \\ \frac{c}{2}\left(\tilde{p}_{t}-\tilde{p}_{t-1}\right)^{2}+\frac{c}{2} \tau\left(\tilde{p}_{t-1}+p^{*}\right)\left(2 \tilde{p}_{t}-\tilde{p}_{t-1}+p^{*}\right)-\tau d-F-B & \text { if } \mu_{t}>0 \wedge \omega_{t} \leq 0 \\ \frac{c}{2}\left(\tilde{p}_{t}-\tilde{p}_{t-1}\right)^{2}-\tau\left(\frac{c}{2}\left(\tilde{p}_{t}+p^{*}\right)^{2}-d-F\right)-F-B & \text { if } \mu_{t} \leq 0 \wedge \omega_{t}>0 \\ \frac{c}{2}\left(\tilde{p}_{t}-\tilde{p}_{t-1}\right)^{2}-F-B & \text { if } \mu_{t} \leq 0 \wedge \omega_{t} \leq 0\end{cases}
$$

with

$\mu_{t}=\frac{c}{2}\left(\tilde{p}_{t-1}+p^{*}\right)\left(2 \tilde{p}_{t}-\tilde{p}_{t-1}+p^{*}\right)-d$

and

$\omega_{t}=\frac{c}{2}\left(\tilde{p}_{t}+p^{*}\right)^{2}-d-F$

The relative fitness function defined in (18) contains four branches. Since $\mu_{t}$ and $\omega_{t}$ represent pre-tax profits of naïve and rational firms, expressed in deviations from the steady state price, the first, second, third and fourth branch imply that all firms make profits, only naïve firms make profits, only rational firms make profits and no firms make profits, respectively. Note that for $\tau=0$, i.e. in the absence of profit taxes, (18) simplifies to $A_{t}=\frac{c}{2}\left(\tilde{p}_{t}-\tilde{p}_{t-1}\right)^{2}-F-B$. If firms furthermore have no preference towards the naïve expectation rule, i.e. if $\tau=0$ and $B=0$, the model corresponds exactly to the model by Brock and Hommes (1997). While fixed costs, represented by parameter $d$, are irrelevant in the original model, they may affect the dynamics of our model, as indicated by the second and third branch of (18).

\section{A number of analytical results}

In this section, we present our analytical results. In section 3.1, we derive the steady state of our model and a necessary and sufficient condition for its local asymptotic stability. In section 
3.2, we examine how the intensity of choice and profit taxes affect the model's steady state and its local asymptotic stability.

\subsection{Steady state and local asymptotic stability}

In general, a firm's steady-state profits may be positive or negative. Since firms cannot permanently sustain losses, from now on we impose the following viability condition

$$
\frac{c}{2}\left(p^{*}\right)^{2}-d-F>0,
$$

which ensures that firms' steady-state profits (with and without tax payments) are positive. As a result, the steady-state value of the rules' relative fitness (18) can be expressed as

$$
A^{*}=-(1-\tau) F-B \leq 0
$$

Under assumption (21), the model's unique steady state is given by

$$
\left(\tilde{p}^{*}, m^{*}\right)=\left(0, \tanh \left(-\frac{1}{2} \beta((1-\tau) F+B)\right)\right)
$$

Recall that a necessary and sufficient condition guaranteeing that a steady state of a twodimensional nonlinear map is locally asymptotically stable is that the two eigenvalues of its Jacobian matrix, calculated at the steady state, are less than one in modulus (see, e.g. Gandolfo 2009 or Medio and Lines 2001). Denoting the eigenvalues by $\lambda_{1}$ and $\lambda_{2}$, the condition for local asymptotic stability can formally be represented by $\left|\lambda_{1,2}\right|<1$. In our case, computation of the eigenvalues reveals that

$$
\lambda_{1}=0
$$

and

$$
\lambda_{2}=-\frac{\left(1-m^{*}\right) c}{\left(1+m^{*}\right) c+2 b} \text {. }
$$

Since $-1 \leq m^{*} \leq 1$, it follows that $\lambda_{2}$ is bounded between $-\frac{c}{b} \leq \lambda_{2} \leq 0$. As in Brock and 
Hommes (1997), we assume that the market is unstable when all firms hold naïve expectations, i.e. demand and supply parameters $b$ and $c$ satisfy that $\frac{c}{b}>1$. Note that $\lambda_{2}=-1$ if $m^{*}=-\frac{b}{c}$. Therefore, local asymptotic stability of the model's steady state requires that $m^{*}>-\frac{b}{c}$.

3.2 Influence of and relation between the intensity of choice and profit taxes

We first explore the role played by the intensity of choice. Let us initially assume that $F>0$, $B>0$ and $0 \leq \tau<1$ so that the steady-state value of the relative fitness of the expectation rules $A^{*}=-((1-\tau) F+B)$ is negative. If the intensity of choice then increases from 0 to $+\infty$, the steady-state difference in market shares $m^{*}$ decreases from 0 to -1 , i.e. the model passes from a situation in which half of the firms hold naïve expectations to a situation in which all firms hold naïve expectations. This can be explained as follows. Since both expectation rules yield identical forecasts at the steady state, the free naïve expectation rule generates higher profits. Moreover, firms have a behavioral preference towards the naïve expectation rule. For some critical value $\beta_{c}$, we obtain $m^{*}=-\frac{b}{c}$ and the steady state becomes unstable. Note that this result holds if $F \geq 0, B>0$ and $0 \leq \tau \leq 1$ or if $F>0, B=0$ and $0 \leq \tau<1$. Suppose first that information costs are zero and/or that the profit tax rate is one. As long as firms have a behavioral preference for the naïve rule, the steady-state value of the rules' relative fitness is negative. Alternatively, if firms have no behavioral preference for the naïve rule, information costs have to be positive and the profit tax rate has to be smaller than one in order to have a negative steady-state value of the relative fitness of the expectation rules. The steady-state value of the relative fitness of the expectation rules is only zero and the model's steady state is 
always locally asymptotically stable, irrespective of the firms' intensity of choice, if $F=0$, $B=0$ and $0 \leq \tau \leq 1$ or if $F \geq 0, B=0$ and $\tau=1$.

What can we say about the role played by profit tax rate $\tau$ ? Here we have to consider three cases. When $F>0$ and $B=0$, referred to as case $X$, the model's steady state simplifies to

$$
X^{*}=\left(\tilde{p}^{*}, m^{*}\right)=\left(0, \tanh \left(-\frac{1}{2} \beta(1-\tau) F\right)\right)
$$

Note first that in this case, which corresponds to the model by Brock and Hommes (1997), augmented by profit taxes, $m^{*}$ increases with $\tau$. Moreover, every destabilizing increase in $\beta$ can be compensated by an increase in $\tau$ such that the steady state remains locally asymptotically stable. For instance, if $\beta$ doubles and $(1-\tau)$ shrinks by 50 percent, $m^{*}$ remains constant. In the extreme scenario in which $\beta=+\infty$, stability of the steady state requires $\tau=1$. Differences in profits or fitness, respectively, are then equal to zero, and the market shares of the two groups are equal. Case $X$ thus has a very strong policy implication. Under the assumptions of case $X$, an unstable steady state can always be stabilized by an increase in the profit tax rate.

When $F=0$ and $B>0$, referred to as case $Y$, our steady state simplifies to

$$
Y^{*}=\left(\tilde{p}^{*}, m^{*}\right)=\left(0, \tanh \left(-\frac{1}{2} \beta B\right)\right)
$$

Obviously, the steady state and its local asymptotic stability are independent of $\tau$. This is because pre-tax profits generated by naïve and rational firms are identical at the steady state and subject to the same profit tax rate, which is why differences in profits are always equal to zero. Accordingly, policy-makers are unable to reestablish market stability by taxing producers' profits. However, the global dynamics of our model is affected by taxes, which we outline in more detail in section 4. 
When $F>0$ and $B>0$, the model's steady state is given by

$Z^{*}=\left(\tilde{p}^{*}, m^{*}\right)=\left(0, \tanh \left(-\frac{1}{2} \beta((1-\tau) F+B)\right)\right)$

which we already defined in (23). In this case, referred to as case $Z$, a destabilizing increase in $\beta$ may be compensated by an increase in $\tau$. Since $\tau$ cannot exceed 1 , stability of the steady state can only be obtained if $\beta \leq \frac{2 \operatorname{arctanh}(b / c)}{B}$. If this condition is satisfied, however, a higher $\tau$ than in case $X$ is needed to obtain stability of the steady state. Suppose that stability in case $X$ is, for a given value of $\beta$, just maintained for $\left(1-\tau^{X}\right) F=V$. For case $Z$, we then have $\left(1-\tau^{Z}\right) F+B=V$. Accordingly, we obtain $\tau^{Z}=\tau^{X}+\frac{B}{F}$. Since $F>0$ and $B>0, \tau^{Z}$ is higher than $\tau^{X}$. What is the economic explanation for this result? Remember that fitness differences in case $X$ are due to profit differences, which can always be leveled by an increasing tax rate so that the steady state remains stable. In case $Z$, however, fitness differences occur not only via profit differences, but also due to parameter $B$. Since the behavioral preference towards naïve expectations is not taxable, profit differences need to be reduced more strongly than in case $X$ in order to obtain the same stability property. This can only be realized by a higher profit tax rate. ${ }^{2}$

\footnotetext{
${ }^{2}$ Suppose that $F>0$ and $B<0$. In this case, say case $Z^{\prime}$, every destabilizing increase in $\beta$ can be compensated by an increase in $\tau$ such that the steady state remains stable. Compared to case $X$, however, a lower $\tau$ is required to obtain stability: since $\tau^{Z^{\prime}}=\tau^{X}+\frac{B}{F}, F>0$ and $B<0$, we have $\tau^{Z^{\prime}}<\tau^{X}$. The explanation for this result is as follows. $B<0$ implies a behavioral preference towards rational expectations. Profit differences, therefore, do not need to be reduced as strongly as in case $X$ to increase the fitness of rational expectations. Branch (2004) argues that individuals have a behavioral preference towards rational expectations. At least with a view to market stability, this may be good news for policy-makers.
} 


\section{A number of numerical results}

In this section, we present a number of simulations to illustrate how the global dynamics of our model depends on the intensity of choice and on profit taxes. In section 4.1, we first explain the general design of our numerical experiments. In sections 4.2, 4.3 and 4.4, we then explore our model's dynamics for cases $X, Y$ and $Z$, respectively.

\subsection{Preliminaries}

Our analytical results provide important insights about how the intensity of choice and the profit tax rate affect the model's steady state and its local asymptotic stability. As we will see, our analytical results also help us to understand the model's global dynamics. To study the global behavior of our model, we consider the following five parameter constellations:

Case $X(H): F=1.0, B=0.0, a=10, b=0.5, c=1.35, d=0.000$

Case $X(L): F=1.0, B=0.0, a=10, b=0.5, c=1.35, d=18.72$

Case $Y(H): F=0.0, B=1.0, a=10, b=0.5, c=1.35, d=0.000$

Case $Y(L): F=0.0, B=1.0, a=10, b=0.5, c=1.35, d=18.72$

Case $Z(H): F=0.8, B=0.2, a=10, b=0.5, c=1.35, d=0.000$

A few comments seem to be in order. We assume in all cases that $F+B=1$. For $\tau=0$, information costs and behavioral bias thus jointly imply the same steady-state fitness advantage of the naïve forecasting rule over the rational forecasting rule. In line with Brock and Hommes (1997), we always assume that $a=10, b=0.5$ and $c=1.35$. As we will see, the global dynamics of our model depends - in contrast to the model's steady state and its local asymptotic stability - on the size of fixed costs $d$. Given $a=10, b=0.5$ and $c=1.35$, steady-state profits generated by naïve and rational firms amount to $19.7224-d$ and 19.7224 $-d-F$, respectively. For $d=18.72$ and, say, $F=1$, steady-state profits generated by naïve and rational firms are still positive and our viability condition is fulfilled. However, 
these steady-state profits are rather low. In fact, once the dynamics starts, profits made by naïve and/or rational firms begin to fluctuate and may occasionally become negative. For $d=0$, steady-state profits made by firms are substantially higher and even remain positive in the presence of larger price fluctuations. ${ }^{3}$ We discuss case $X$ and case $Y$ when firms' steadystate profits are high and low. For case $Z$, it is sufficient to focus on the scenario when firms' steady-state profits are high. In total, this generates five cases: $X(H), X(L), Y(H), Y(L)$ and $Z(H)$, where $H$ and $L$ stand for high and low steady-state profits, respectively.

To analyze the effect of both the intensity of choice and the profit tax rate on our model dynamics, we use $\beta$ and $\tau$ as bifurcation parameters. In all bifurcation diagrams, the bifurcation parameter is increased in 500 discrete steps, while all other parameters remain constant. For each parameter value, we plot 30 observations after erasing a transient phase of 1000 periods. For some parameter combinations, we present basins of attraction of the steady state and other coexisting attractors. For these computations, a transient phase of 1000 periods has been omitted. To investigate whether our results are robust, we occasionally add exogenous noise to the dynamics. In these experiments, we then add a normally distributed random variable with mean zero and standard deviation 0.10 to the evolution of $\tilde{p}_{t}$ in (16).

To be able to quantify the effect of profit taxes on our model dynamics, we introduce two statistics. As a measure of the variability of prices, we define volatility $=\frac{1}{T} \sum_{t=1}^{T}\left(\frac{p_{t}-p_{t-1}}{p_{t-1}}\right)^{2}$;

to identify the market's mispricing we use

\footnotetext{
${ }^{3}$ To be precise, the value of parameter $a$ is irrelevant in the model by Brock and Hommes (1997). In our model, parameter $a$ may render a firm's profits positive or negative and thus for $\tau>0$ it has an impact on its global dynamics. Since it is more intuitive to control the size of a firm's profits via parameter $d$, we keep parameter $a$ constant at $a=10$.
} 
distortion $=\frac{1}{T} \sum_{t=1}^{T}\left(\frac{p_{t}-p^{*}}{p^{*}}\right)^{2}$,

where $T$ represents the length of the underlying sample. When we compute these statistics, we add the aforementioned exogenous noise to the dynamics, omit a transient phase of 1000 periods and use a sample length of $T=5000$ periods.

\subsection{The dynamics of the model in case $X$}

We start our numerical analysis with case $X(H)$, i.e. we set $F=1, B=0$ and $d=0$. Under these assumptions, our model corresponds to that by Brock and Hommes (1997), augmented by profit taxes. Figure 1 provides a number of examples about how the intensity of choice and profit taxes may affect the model dynamics. Panel (a) presents a bifurcation diagram for the intensity of choice. Since the profit tax rate is set to $\tau=0$, we face exactly the same scenario explored in Brock and Hommes (1997). ${ }^{4}$ As can be seen, the bifurcation route evolves from a stable steady state to chaotic price fluctuations as $\beta$ increases from 0 to 5 . The primary bifurcation towards instability is a period-doubling bifurcation at which the steady state becomes unstable and a stable 2-cycle emerges. If $\beta$ becomes larger, further bifurcations occur and the model dynamics becomes increasingly complicated. To explore the effect of profit taxes, we repeat our simulations from panel (a) in panel (b), but now setting $\tau=0.5$. The corresponding bifurcation route shows that the primary bifurcation occurs for a higher value of the intensity of choice (as can also be verified analytically, $\beta_{c}$ doubles from $\beta_{c} \approx 0.78$ to $\beta_{c} \approx 1.56$ ). Moreover, price fluctuations are more dampened (and less complicated) for high values of the intensity of choice.

[Figure 1 about here]

\footnotetext{
${ }^{4}$ See, for instance, figure 5.2 on page 142 in Hommes (2013).
} 
The stabilizing impact of profit taxes can be identified most clearly from panel (c) of figure 1 , which presents a bifurcation diagram for $\tau$ with $\beta=5$. By increasing the profit tax rate from 0 to 1 , we observe that chaotic price fluctuations eventually converge to $\tilde{p}^{*}=0$. To be precise, the model's steady state becomes stable when the profit tax rate exceeds $\tau=0.844$. Again, this observation is in line with our analytical results as $(1-0.844) 5$ yields 0.78, the value of $\beta_{c}$ for $\tau=0$. A closer comparison of panels (a) and (c) reveals that the rational route to randomness from panel (a) can be reverted exactly by increasing the tax rate from 0 to 1 . The explanation for this - at least at first sight - puzzling result is surprisingly simple. Under parameter setting $X(H)$, firms always make profits. Therefore, only the first branch of the relative fitness function (18) matters, and (17) and (18) can be combined to $m_{t}=\tanh \left(\frac{1}{2} \beta(1-\tau)\left(\frac{C}{2}\left(\tilde{p}_{t}-\tilde{p}_{t-1}\right)^{2}-F\right)\right)$. We immediately see that every destabilizing increase in $\beta$ can be compensated by an appropriate increase in $\tau$. For instance, a doubling of $\beta$ necessitates a halving of $(1-\tau)$ to keep the term $\beta(1-\tau)$ constant. Panel (d) repeats our simulation from panel (c) in a noisy environment. As it turns out, the stabilizing effect of profit taxes is robust with respect to additional exogenous noise - at least with a view to the amplitude of price fluctuations. Panels (e) and (f) in figure 1 show how our policy measures react to increasing tax rates. As in panel (d), we set $\beta=5$ and add exogenous noise to the dynamics. Overall, volatility and distortion decrease with increasing profit tax rates. The reduction in volatility is only slowed down due to high-amplitude noisy cycles for an intermediate range of profit tax rates, say between 0.4 and 0.6 .

How does the dynamics change if firms' profits are not always positive? Figure 2 illustrates the dynamics of the model for case $X(L)$ with $F=1, B=0$ and $d=18.72$. Since the design of figure 2 is identical to that of figure 1, our simulations can be compared directly with each other. The profit tax rate in panel (a) is set to $\tau=0$ so that fixed costs are 
irrelevant. As a result, the bifurcation diagram in panel (a) of figure 2 is equivalent to the bifurcation diagram in panel (a) of figure 1. Accordingly, an increase in the intensity of choice again triggers a rational route to randomness. In panel (b) we repeat these simulations for $\tau=0.5$ and also observe for case $X(L)$ a stabilizing effect of profit taxes. Compared to panel (b) of figure 1, however, two aspects deserve our attention. First, in case $X(L)$ the 2-cycle emerges abruptly with a significant amplitude and not smoothly, as in case $X(H)$. We investigate this intriguing phenomenon, caused by the emergence of coexisting attractors, in more detail in figure 3. Second, while profit taxes also stabilize the dynamics in case $X(L)$, the effect is less powerful than in case $X(H)$.

[Figure 2 about here]

The bifurcation diagram in panel (c) of figure 2 also reveals that an increasing tax rate decreases the amplitude of price fluctuations. Moreover, a convergence to the steady state sets in again when $\tau$ exceeds 0.84 . However, the bifurcation route differs from the bifurcation route in panel (c) of figure 1. In particular, the stabilizing effect of increasing profit taxes is much weaker. Furthermore, panel (c) of figure 2 also suggests that the steady state may coexist for some values of $\tau$ with other types of attractors (which we will also discuss in figure 3). In panel (d), we observe that the stabilizing effect of an increasing profit tax rate holds with respect to exogenous noise which, in turn, is further supported by panels (e) and (f). Both volatility and distortion decrease smoothly as the profit tax rate increases from 0 to 1 , although the effect is weaker than in case $X(H)$.

The bifurcation diagram in panel (b) of figure 2 is based on initial conditions which are close to the model's steady state. In contrast, the bifurcation diagram depicted in panel (a) of figure 3 is based on initial conditions which are more distant from the model's steady state. A comparison of these bifurcations diagrams reveals the coexistence of different attractors, 
approximately between $1.12<\beta<1.56 .{ }^{5}$ Indeed, in panel (c) of figure 3 , we plot time series of our model for different initial values when $\beta=1.2$ and $\tau=0.5$. Obviously, one orbit turns into a 2-cycle (black line, generated with $\tilde{p}_{1}=0.25$ and $m_{1}=m^{*}$ ) while the other converges to the model's steady state (red line, generated with $\tilde{p}_{1}=0.1$ and $m_{1}=m^{*}$ ). The corresponding basins of attraction are visualized in panel (e). For initial values included in the light red area, the system converges to its steady state (represented by the red dot). Initial values from the light gray area imply a convergence to a 2-cycle (represented by black dots).

Coexisting attractors may have interesting policy implications. Suppose that the price has converged towards the model's steady state. As long as exogenous shocks are not too large, the system will not leave the steady state's basin of attraction, and endogenous forces will drive the price back towards its equilibrium value. However, if the exogenous shocks are larger, the system may be pushed into the light gray area and the price then endogenously starts to fluctuate up and down. Panel (g) reveals that the light red area increases with the profit tax rate (simulations are based on $\tau=0.585$ ). Policy-makers may thus have the chance to drive back the price to its steady state by (temporarily) increasing the profit tax rate. In contrast, a reduction in the profit tax rate or an increase in the intensity of choice (not depicted) reduces the steady state's basin of attraction.

[Figure 3 about here]

\footnotetext{
${ }^{5}$ Let us be more precise. At about $\beta=1.12$, we observe a fold border collision bifurcation, i.e. the birth of an attracting and a repelling 2-cycle. The transition between the steady state and the attracting 2-cycle is sharp: when the attracting 2-cycle emerges it is already distant to the steady state and reached from a larger set of initial conditions. As $\beta$ increases, the amplitude of the attracting 2-cycle increases while the amplitude of the repelling 2-cycle decreases. At about $\beta=1.56$, the repelling 2-cycle merges with the steady state and we witness a subcritical flip bifurcation. A deeper analysis of this bifurcation structure is possible, but beyond the scope of our paper. However, more details are available upon request. We greatly thank Irina Sushko for helping us to understand what is occurring here.
} 
A similar phenomenon can be detected by comparing the bifurcation diagrams in panel (c) of figure 2 and in panel (b) of figure 3. Both bifurcation diagrams are based on $\beta=5$ and $0 \leq \tau \leq 1$. Between $0.84 \leq \tau \leq 1$, the steady state coexists with other types of attractors. One example is given in panel (d) of figure 3, which shows trajectories for two different initial values based on $\beta=5$ and $\tau=0.85$. While our model produces chaotic dynamics for $\tilde{p}_{1}=0.25$ and $m_{1}=m^{*}$ (black line), the price converges to its steady state for $\tilde{p}_{1}=0.001$ and $m_{1}=m^{*}$ (red line). Panel (f) visualizes the coexisting attractors' basins of attraction. In this example, the basin of attraction of the steady state is rather small. Further simulations reveal that the steady state also coexists with different cycles as the profit tax rate increases. For instance, for $\tau=0.95$ the steady state coexists with an 8-cycle while for $\tau=1$ the steady state coexists with a 4-cycle. ${ }^{6}$ Panel (h) contains an example for $\tau=0.99$. It should be noted that the amplitude of cycles decreases with the profit tax rate and that the basin of attraction of the coexisting steady state increases with the profit tax rate. Also from this perspective, higher profit tax rates may be regarded as beneficial for market stability.

Let us briefly summarize our numerical results for case $X$. Due to information costs, naïve firms make (on average) higher profits than rational firms. As the intensity of choice increases, more and more firms thus switch to naïve expectations and the amplitude of price fluctuations increases. By imposing profit taxes, policy-makers have the opportunity to reduce profit differences between naïve and rational expectations. If they do this, more firms will rely on rational expectations, and the price dynamics becomes dampened. The stabilizing effect of profit taxes is particularly strong if both types of firms continuously generate profits. Naturally, if a firm's profits are negative, it does not have to pay taxes. The more frequently

\footnotetext{
${ }^{6}$ Of course, technically, we can raise $\tau$ above one. In doing so, we first observe that the 4-cycle turns into a 2cycle before the dynamics eventually converges to the model's steady state. It seems that the 2-cycle emerges again due to a fold border collision bifurcation.
} 
the firm's profits are negative, the lower the stabilizing effect of profit taxes. We have also seen that the dynamics of our model may give rise to coexisting attractors. Numerical evidence suggests that the steady state's basin of attraction increases with the profit tax rate while the amplitude of the fluctuations of the coexisting attractor decreases simultaneously. Overall, policy-makers may thus want to increase the profit tax rate in case $X$ to stabilize the dynamics.

\subsection{The dynamics of the model in case $Y$}

Let us turn to case $Y(H)$ and assume that $F=0, B=1$ and $d=0$. Panel (a) of figure 4 shows a bifurcation diagram for the intensity of choice. Since $\tau=0$ and $F+B=1$, we observe for increasing values of the intensity of choice the same rational route to randomness as in panel (a) of figure 1 and in panel (a) of figure 2. In panel (b) of figure 4, we repeat the simulation from panel (a) for $\tau=0.5$. Note that the steady state and its local asymptotic stability are independent of the profit tax while the model's global behavior is clearly affected by it. As can be seen, the primary bifurcation towards instability occurs again at $\beta_{C} \approx 0.78$. However, once the intensity of choice exceeds this value, the price dynamics shows higher amplitude fluctuations than without profit taxes. For instance, for $\beta=5$ and $\tau=0$ price fluctuations are bounded between \pm 1.29 while for $\beta=5$ and $\tau=0.5$ they scatter between \pm 1.83 .

To illustrate these results in more detail, we present two bifurcation diagrams in which we vary the profit tax rate between 0 and 1 . In panel (c), we set $\beta=5$ and thus the model produces complex dynamics for $\tau=0$. In panel (g), we assume that $\beta=0.75$ so that the model's steady state is locally asymptotically stable for $\tau=0$. The bifurcation route in panel (c) undoubtedly reveals that higher profit taxes amplify price fluctuations. As depicted in panel (d), this is also true in a noisy environment. Further evidence of this result is provided 
by panels (e) and (f), which reveal that volatility and distortion grow with $\tau$. In panel (g), the dynamics always converges towards $\tilde{p}^{*}=0$, irrespective of the profit tax rate. Nevertheless, a destabilizing impact of profit taxes can be observed when the simulation from panel (g) is repeated with exogenous noise. The increase in the amplitude of the price fluctuations in panel (h) is also confirmed by panels (i) and (j). Both volatility and distortion slope upwards as the profit tax rate increases. While the destabilizing effect of profit taxes seems to be rather weak in absolute terms, volatility and distortion more than triple in relative terms.

[Figure 4 about here]

How do profit taxes influence the dynamics of our model in case $Y(H)$ ? Let us begin with the scenario depicted in panel (g) of figure 4 in which the steady state is locally asymptotically stable. In the absence of information costs $(F=0)$, firms using the naïve or the rational expectation rule realize identical pre-tax profits. Since both types of firms are subject to the same profit tax rate, differences in profits are equal to zero and therefore independent of $\tau$. If we exogenously stimulate the dynamics by adding noise, the picture starts to change. In the presence of price fluctuations, rational expectations deliver more precise predictions than naïve expectations and are thus more profitable. If these profit differences are taxed away, fewer firms opt for rational expectations, and price fluctuations increase. The explanation for what is occurring in panel (c) is similar. Recall that firms have a behavioral preference towards the simple expectation rule, which is why more and more firms use naïve expectations as the intensity of choice increases. When prices start to fluctuate, rational expectations outperform naïve expectations. However, the economic fitness advantage of rational expectations decreases with the profit tax rate. Since the behavioral fitness advantage of naïve expectations is not taxable, firms switch from rational expectations to naïve expectations as the profit tax rate increases and, therefore, price fluctuations amplify. 
Clearly, policy-makers who intend to stabilize the dynamics should promote rational expectations by decreasing profit taxes.

Figure 5 illustrates our model dynamics for case $Y(L)$. For $F=0, B=1$ and $d=18.72$, firms' steady-state profits are still positive, yet at a much lower level than before. As a result, out-of-equilibrium profits of firms may, at least temporarily, become negative. To be able to draw direct comparisons between cases $Y(L)$ and $Y(H)$, figure 5 has the same design as figure 4. In panel (a) of figure 5, we observe our standard rational route to randomness. When we set $\tau=0.5$, the bifurcation route from panel (a) changes to that presented in panel (b). Again, the steady state and its local asymptotic stability are not influenced by the imposition of profit taxes, but the model's global behavior is. Note that in comparison to panel (b) of figure 4, price dynamics is less destabilized by increasing profit tax rates. The bifurcation route for the profit tax rate in panel (c) reveals that price fluctuations amplify with $\tau$. Panels (d), (e) and (f) demonstrate that this observation is robust with respect to exogenous noise. However, the effect is much weaker than in case $Y(H)$. Panel (g) reveals that the destabilizing impact of an increase in $\tau$ is also weaker when $\beta=0.75$. This finding also holds in a noisy environment, as is witnessed in panels (h), (i) and (j). In the (unrealistic) limit in which firms always make losses, profit taxes obviously become irrelevant for the model dynamics.

[Figure 5 about here]

Let us summarize our numerical results for case $Y$. Due to firms’ behavioral bias towards naïve expectation rules, more and more firms abstain from rational expectations as the intensity of choice increases. Consequently, the model's steady state becomes unstable and endogenous dynamics set in. When policy-makers impose profit taxes, the profit advantage of rational expectations decreases while the behavioral advantage of naïve expectations remains constant. This leads to fewer firms relying on rational expectations and 
less stable price dynamics. To foster market stability, policy-makers may wish to promote rational expectations by decreasing profit taxes. Overall, the destabilizing impact of profit taxes depends on both the level of the intensity of choice and the level of steady-state profits. If the intensity of choice is low, firms do not realize fitness differentials very well. Policymakers can then only moderately affect the behavior of firms by changing the profit tax rate. If steady-state profits are low, price fluctuations may drive firms' profits into the negative zone. The more frequently firms make losses, the less effectively policy-makers can use profit taxes to control the fitness difference between naïve and rational expectations.

\subsection{The dynamics of the model in case $Z$}

Before we begin with our numerical analysis of case $Z$, let us briefly contrast the key results of cases $X$ and $Y$. In case $X$, free naïve expectations generate (on average) higher profits than costly rational expectations. Policy-makers seeking to calm down price fluctuations thus have to curb naïve expectations by increasing profit taxes. In case $Y$, rational expectations are free and outperform naïve expectations, but firms have a behavioral preference towards naïve expectations. To improve market stability, policy-makers now have to increase the popularity of rational expectations by reducing profit taxes. Case $Z$ may be regarded as a nontrivial combination of cases $X$ and $Y$. In case $Z$, rational expectations are costly, and firms have a behavioral preference towards naïve expectations. As we will see, there are parameter constellations in which the basic results of case $X$ survive, in which the basic results of case $Y$ survive and in which a mix of the results of cases $X$ and $Y$ appears.

Figure 6 illustrates how profit taxes may affect the dynamics of our model under the scenario of case $Z(H)$, i.e. for $F=0.8, B=0.2$ and $d=0 .{ }^{7}$ Since $F+B=1$ and $\tau=0$, the bifurcation diagram with respect to the intensity of choice, depicted in panel (a), portrays our standard rational route to randomness. In panel (b), we repeat the simulation from panel (a).

\footnotetext{
${ }^{7}$ Since case $Z(L)$ yields no additional results, it is omitted for the sake of brevity.
} 
For $\tau=0.5$, we observe a stabilizing effect of profit taxes: the primary bifurcation shifts from $\beta_{c} \approx 0.78$ to $\beta_{c} \approx 1.30$ and, for a given value of $\beta$, price fluctuations are lower. How do these results relate to case $X(H)$ and case $Y(H)$ ? Compared to case $X(H)$, the primary bifurcation towards instability already occurs at $\beta_{C} \approx 1.30$ instead of $\beta_{C} \approx 1.56$, and the price dynamics is, for a given value of $\beta$, more volatile (see figure 1, panel (b)). The explanation of why profit taxes work less effectively in case $Z(H)$ than in case $X(H)$ is as follows. Fitness differences in case $X(H)$ result from profit differences only, while in case $Z(H)$ they also contain a (tax-independent) behavioral component. As a result, the fitness difference is not reduced as strongly as in case $X(H)$ when profit taxes are imposed. Put differently, to obtain the same fitness difference as in case $X(H)$, profit differences have to be reduced more strongly. As revealed by our analytical results, this can be achieved by imposing a higher tax rate. For instance, policy-makers have to increase the profit tax rate from $\tau=0.5$ to $\tau=0.625$ to elevate the primary bifurcation from $\beta_{C} \approx 1.30$ to $\beta_{C} \approx 1.56$. Recall that in case $Y(H)$, profit taxes do not affect the properties of the steady state, but are destabilizing once endogenous dynamics kick in (see figure 4, panel (b)).

[Figure 6 about here]

Whether profit taxes have to be increased or decreased to improve market stability depends, amongst others, on the intensity of choice and the associated price variability. To exemplify this point, we choose three different values for the intensity of choice. In panels (c), (e) and (g) we increase $\tau$ from 0 to 1 and set $\beta=5, \beta=3$ and $\beta=25$, respectively. Panels (d), (f) and (h) show the corresponding bifurcation routes when exogenous noise is added. Let us begin with panel (e) in which the model dynamics eventually converges to $\tilde{p}^{*}=0$ as $\tau$ increases. This can be explained as follows. For $\tau=0$, price fluctuations are rather moderate. Therefore, prediction errors of naïve expectations are relatively low and the simple 
forecasting strategy is the more profitable one. When policy-makers impose profit taxes, the effect of reducing profit differences dominates the behavioral bias towards naïve expectations, and the dynamics of our model stabilizes. As can be checked analytically, for $\beta=3$ the model's steady state becomes stable when the profit tax rate crosses $\tau=0.93$. However, the profit tax rate cannot exceed 1 and stability of the steady state can therefore only be obtained in case $Z$ if $\beta \leq \frac{2 \operatorname{arctanh}(b / c)}{B} \leq 3.89$. From panel (g), in which $\beta=25$, we observe that price fluctuations increase with $\tau$. Note that for $\beta=25$ and $\tau=0$, price fluctuations are so strong that it is (often) beneficial to buy rational expectations. The imposition of profit taxes thus penalizes the use of rational expectations more strongly than the use of naïve expectations. Since the behavioral fitness advantage is independent of $\tau$, naïve expectations gain in popularity as profit taxes increase and, consequently, price dynamics becomes even more unstable. One may argue that the dynamics depicted in panel (e) is basically in line with the results we obtain in case $X$ while the dynamics shown in panel (g) is basically in line with the results we obtain in case $Y$. The dynamics visualized in panel (c) may be regarded as a nontrivial combination of these results. We first observe that an increase in the profit tax rate decreases the amplitude of price fluctuations before price fluctuations start to increase again. Panels (d), (f) and (h) reveal that our observations also hold in a noisy environment. The corresponding volatility and distortion functions (not depicted) further support our findings.

Let us summarize our numerical results for case $Z(H)$. Since we assume that rational expectations are costly and that firms have a behavioral preference towards naïve expectations, more and more firms rely on naïve expectations as the intensity of choice increases and, as a result, the model's dynamics becomes increasingly complicated. Whether policy-makers need to increase or decrease profit taxes to foster market stability depends, amongst others, on the firm's intensity of choice. If the intensity of choice is low, prices fluctuate only mildly and simple expectations generate higher profits. When policy-makers 
impose profit taxes in such a situation, the effect of reducing the profit advantage of naïve expectations over rational expectations dominates the behavioral fitness advantage of naïve expectations. More firms then rely on rational expectations, and price dynamics are more stable. If the intensity of choice is high, prices fluctuate strongly and, consequently, rational expectations are more profitable than naïve expectations. Policy-makers are able to further promote rational expectations and thus to stabilize price fluctuations by decreasing profit taxes. For some parameter constellations, however, we observe that an increase in the profit tax rate initially stabilizes the dynamics, but then destabilizes it.

\section{Conclusions}

The dynamic behavior of many markets depends crucially on the expectations of their market participants. Empirical evidence reveals that market participants use different rules to form their expectations. As a result, the dynamic behavior of a market depends on the mix of expectation rules applied. For instance, a market may be rather stable if stabilizing expectation rules are more popular than destabilizing expectation rules. Empirical evidence furthermore indicates that the market participants' selection of expectation rules depends on economic fitness criteria such as profits realized in the past. In line with these observations, Brock and Hommes (1997) develop a cobweb model in which firms adapt their price expectations by a profit-based switching between free naïve expectations and costly rational expectations. One of their key results is that fixed-point dynamics may turn into increasingly complex dynamics as the firms' intensity of choice (i.e. their response to fitness differences) increases. The main contribution of our paper is to show that policy-makers may be able to manage such rational routes to randomness. In particular, we find that policy-makers should increase (decrease) profit taxes if destabilizing expectation rules generate higher (lower) profits than stabilizing expectation rules. This alters the composition of expectation rules 
applied in favor of more stabilizing expectation rules and thus calms down a market's price fluctuations.

To make the exposition of our arguments as clear as possible, we use the seminal cobweb model by Brock and Hommes (1997) as a simple, yet powerful reference model. Our analysis may be extended in various directions. For instance, one may equip firms with different and/or more expectation rules to forecast futures prices. Policy-makers would then have to determine whether the more stabilizing or more destabilizing expectation rules produce higher profits. As revealed by our analysis of case $Z(H)$, there may be a nontrivial relation between market stability and profit tax rates. Identifying volatility-minimizing profit tax rates may be a challenging task in reality. One may also substitute the discrete choice approach of Manski and McFadden (1981) with another switching model, i.e. the transition probability approach of Weidlich and Haag (1983). Alternatively, one could think about investigating the stabilizing effects of other tax measures. For example, what are the consequences of revenue taxes, lump sum taxes or subsidies? Moreover, the model by Brock and Hommes (1997) is a partial equilibrium model. How do our results change in a general equilibrium framework? In our setup, tax revenues are not redistributed and our focus is on market stability. Against this backdrop, a welfare analysis may be worthwhile. Instead of exploring the dynamics of cobweb markets, one could also study different markets, say financial markets in which traders have to pay taxes on their speculative profits.

Nevertheless, our paper reveals that, as long as the market participants' selection of their expectation rules depends on economic fitness criteria, policy-makers can affect the mix of the expectation rules applied promoting market stability. Given the volatility of real markets, we regard this as an important insight. 


\section{References}

Anufriev, M., and Panchenko, V. (2009): Asset prices, traders' behavior and market design. Journal of Economic Dynamics and Control, 33, 1073-1090.

Anufriev, M., and Hommes, C. (2012): Evolutionary selection of individual expectations and aggregate outcomes in asset pricing experiments. American Economic Journal: Microeconomics, 4, 35-64.

Anufriev, M. and Tuinstra, J. (2013a): The impact of short-selling constraints on financial market stability in a heterogeneous agents model. Journal of Economic Dynamics and Control, 37, 1523-1543.

Anufriev, M., Bao, T. and Tuinstra, J. (2013b): Fund choice behavior and estimation of switching models: an experiment. CeNDEF Working Paper 13-04, University of Amsterdam.

Anufriev, M., Assenza, T., Hommes, C. and Massaro, D. (2013): Interest rate rules and macroeconomic stability under heterogeneous expectations. Macroeconomic Dynamics, 17, 1574-1604.

Baak, S. (1999): Tests for bounded rationality with a linear dynamic model distorted by heterogeneous expectations. Journal of Economic Dynamics and Control, 23, 1517-1543.

Boswijk, P., Hommes, C. and Manzan, S. (2007): Behavioral heterogeneity in stock prices. Journal of Economic Dynamics and Control, 31, 1938-1970.

Branch, W. (2004): The theory of rationally heterogeneous expectations: Evidence from survey data on inflation expectations. The Economic Journal, 114, 592-621.

Branch, W. and McGough, B. (2008): Replicator dynamics in a cobweb model with rationally heterogeneous expectations. Journal of Economic Behavior and Organization, 65, 224-244.

Branch, W. and McGough, B. (2010): Dynamic predictor selection in a new Keynesian model with heterogeneous expectations. Journal of Economic Dynamics and Control, 34, 14921508.

Brock, W. and Hommes, C. (1997): A rational route to randomness. Econometrica, 65, 10591095.

Brock, W. and Hommes, C. (1998): Heterogeneous beliefs and routes to chaos in a simple asset pricing model. Journal of Economic Dynamics Control, 22, 1235-1274.

Brock, W., Hommes, C. and Wagener, F. (2005): Evolutionary dynamics in markets with many trader types. Journal of Mathematical Economics, 41, 7-42.

Brock, W., Hommes, C. and Wagener, F. (2009): More hedging instruments may destabilize markets. Journal of Economic Dynamics and Control, 33, 1912-1928.

Chavas, J. (2000): On information and market dynamics: The case of the U.S. beef market. Journal of Economic Dynamics and Control, 24, 833-853. 
Chiarella, C., Dieci, R. and He, X.-Z. (2009): Heterogeneity, market mechanisms, and asset price dynamics. In: Hens, T. and Schenk-Hoppé, K.R. (eds.): Handbook of Financial Markets: Dynamics and Evolution. North-Holland, Amsterdam, 277-344.

Chiarella, C., Dieci, R., He, X.-Z. and Li, K. (2013): An evolutionary CAPM under heterogeneous beliefs. Annals of Finance, 9, 185-215.

De Grauwe, P. (2011): Animal spirits and monetary policy. Economic Theory, 47, 423-457.

De Grauwe, P. and Grimaldi, M. (2006): Exchange rate puzzles: A tale of switching attractors. European Economic Review, 50, 1-33.

Dick, C. and Menkhoff, L. (2013): Exchange rate expectations of chartists and fundamentalists. Journal of Economic Dynamics and Control, 37, 1362-1383.

Dieci, R. and Westerhoff, F. (2014): Heterogeneous expectations, boom-bust housing cycles, and supply conditions: a nonlinear dynamics approach. Working Paper, University of Bamberg.

Diks, C. and van der Weide, R. (2003): Heterogeneity as a natural source of randomness. Tinbergen Institute Discussion Paper, No. 03-073/1.

Diks, C. and van der Weide, R. (2005): Herding, a-synchronous updating and heterogeneity in memory in a CBS. Journal of Economic Dynamics and Control, 29, 741-763.

Droste, E., Hommes, C. and Tuinstra, J. (2002): Endogenous fluctuations under evolutionary pressure in Cournot competition. Games and Economic Behavior, 40, 232-269.

Franke, R. and Westerhoff, F. (2012): Structural stochastic volatility in asset pricing dynamics: estimation and model contest. Journal of Economic Dynamics and Control, 36, 1193-1211.

Gandolfo, G. (2009): Economic dynamics. Springer, Heidelberg.

Goeree, J. and Hommes, C. (2000): Heterogeneous beliefs and the non-linear cobweb model. Journal of Economic Dynamics and Control, 24, 761-798.

Goldbaum, D. and Zwinkels, R. (2013): An empirical examination of heterogeneity and switching in foreign exchange markets. Journal of Economic Behavior and Organization, in press.

Hommes, C. (2011): Behavioral rationality and heterogeneous expectations in complex economic systems. Cambridge University Press, Cambridge.

Hommes, C. and Wagener, F. (2009): Complex evolutionary systems in behavioral finance. In: Hens, T. and Schenk-Hoppé, K.R. (eds.): Handbook of Financial Markets: Dynamics and Evolution. North-Holland, Amsterdam, 217-276.

Hommes, C., Huang, H. and Wang, D. (2005): A robust rational route to randomness in a simple asset pricing model. Journal of Economic Dynamics and Control, 29, 1043-1072.

Kindleberger, C. (2000): Manias, panics and crashes: A history of financial crises. Wiley, 
New York.

Kouwenberg, R. and Zwinkels, R. (2014): Forecasting the US housing market. International Journal of Forecasting, 30, 415-425.

Lasselle, L., Svizzero, S. and Tisdell, C. (2005): Stability and cycles in a cobweb model with heterogeneous expectations. Macroeconomic Dynamics, 9, 630-650.

Lines, M. and Westerhoff, F. (2010): Inflation expectations and macroeconomic dynamics: The case of rational versus extrapolative expectations. Journal of Economic Dynamics and Control, 34, 246-257.

Lux, T. (2009): Stochastic behavioural asset-pricing models and the stylized facts. In: Hens, T. and Schenk-Hoppé, K.R. (eds.): Handbook of Financial Markets: Dynamics and Evolution. North-Holland, Amsterdam, 161-216.

Manski, C. and McFadden, D. (1981): Structural analysis of discrete data with econometric applications. MIT Press, Cambridge.

Menkhoff, L. and Taylor, M. (2007): The obstinate passion of foreign exchange professionals: technical analysis. Journal of Economic Literature, 45, 936-972.

Medio, A. and Lines, M. (2001): Nonlinear dynamics: A primer. Cambridge University Press, Cambridge.

Reinhart, C. and Rogoff, K. (2009): This time is different: Eight centuries of financial folly. Princeton University Press, Princeton.

Shiller, R. (2005): Irrational exuberance. Princeton University Press, Princeton.

Ter Ellen, S. and Zwinkels, R. (2010): Oil price dynamics: A behavioral finance approach with heterogeneous agents. Energy Economics, 32, 1427-1434.

Tuinstra, J., Wegener, M. and Westerhoff, F. (2014): Positive welfare effects of trade barriers in a dynamic partial equilibrium model. Journal of Economic Dynamics and Control, in press.

Weidlich, W. and Haag, G. (1983): Concepts and models of a quantitative sociology. Springer, Berlin.

Westerhoff, F. and Dieci, R. (2006): The effectiveness of Keynes-Tobin transaction taxes when heterogeneous agents can trade in different markets: a behavioral finance approach. Journal of Economic Dynamics and Control, 30, 293-322.

Westerhoff, F. and Franke, R. (2014): Agent-based models for economic policy design: two illustrative examples. In: Chen, S.-H., Kaboudan, M. and Du, Y,-R. (eds.): OUP Handbook on Computational Economics and Finance. Oxford University Press, Oxford, in press.

Yeh, C. and Yang, C. (2010): Examining the effectiveness of price limits in an artificial stock market. Journal of Economic Dynamics and Control, 34, 2089-2108. 
(a)

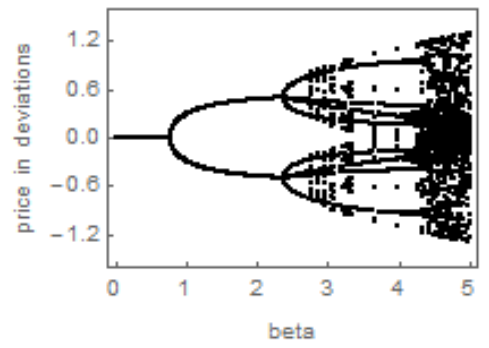

(c)

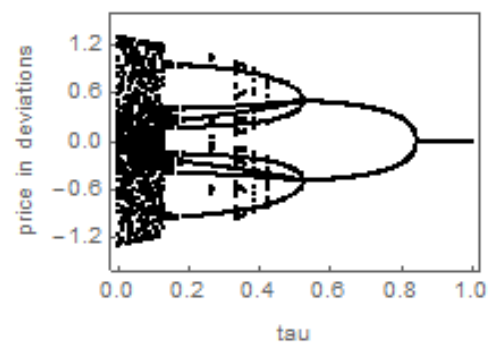

(e)

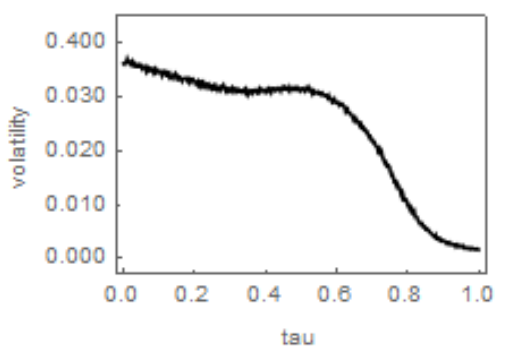

(b)

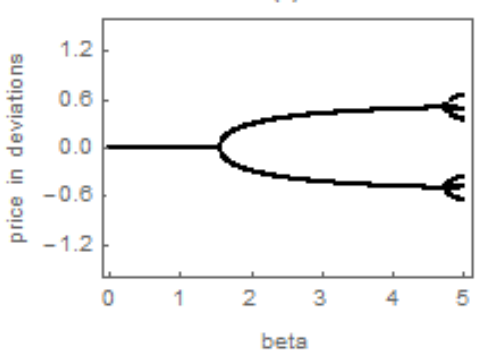

(d)

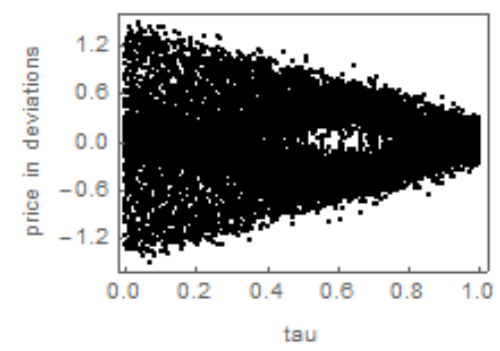

(f)

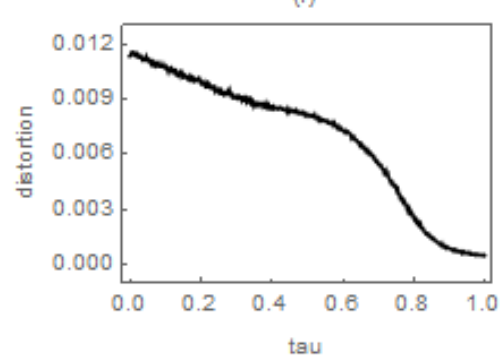

Figure 1: Dynamics for case $X(H)$. (a) Bifurcation diagram for the intensity of choice with $\tau=0$. (b) Bifurcation diagram for the intensity of choice with $\tau=0.5$. (c) Bifurcation diagram for the profit tax rate with $\beta=5$. (d) Bifurcation diagram for the profit tax rate with $\beta=5$ and exogenous noise. (e) Volatility as a function of profit taxes with $\beta=5$ and exogenous noise. (f) Distortion as a function of profit taxes with $\beta=5$ and exogenous noise. 
(a)

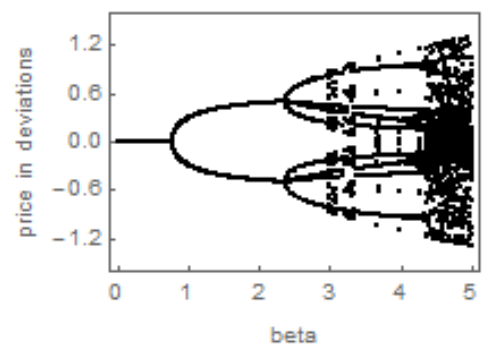

(c)

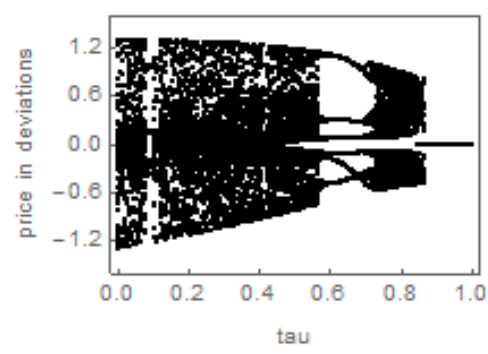

(e)

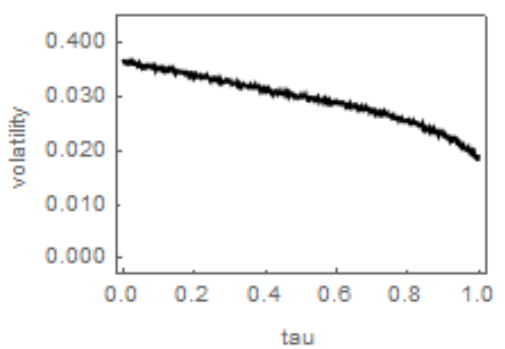

(b)

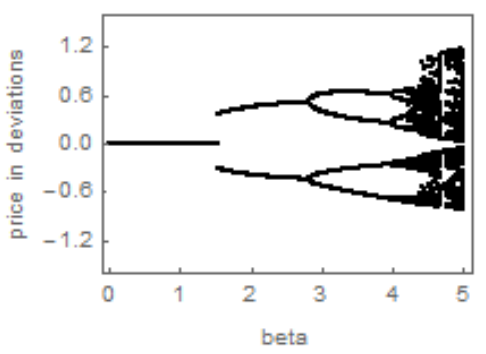

(d)

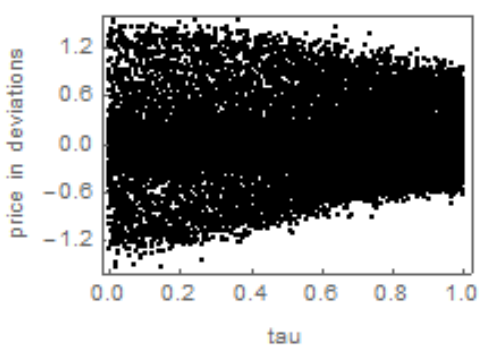

(f)

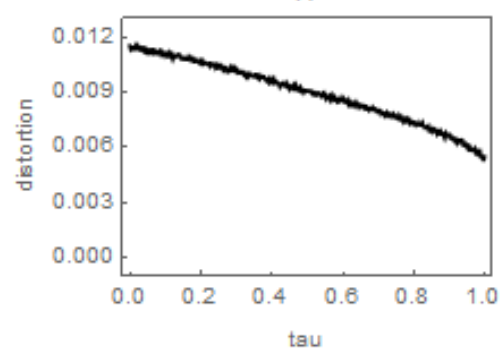

Figure 2: Dynamics for case $X(L)$. (a) Bifurcation diagram for the intensity of choice with $\tau=0$. (b) Bifurcation diagram for the intensity of choice with $\tau=0.5$. (c) Bifurcation diagram for the profit tax rate with $\beta=5$. (d) Bifurcation diagram for the profit tax rate with $\beta=5$ and exogenous noise. (e) Volatility as a function of profit taxes with $\beta=5$ and exogenous noise. (f) Distortion as a function of profit taxes with $\beta=5$ and exogenous noise. 
(a)

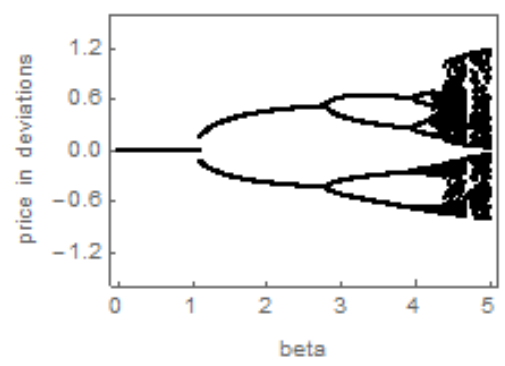

(c)

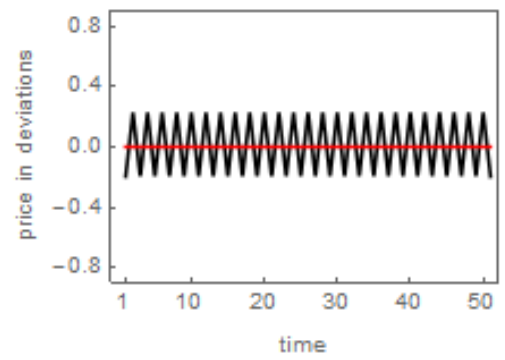

(e)

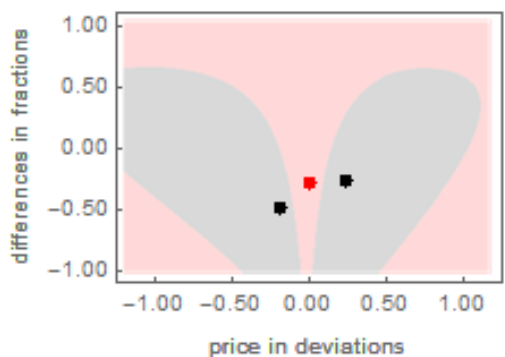

(g)

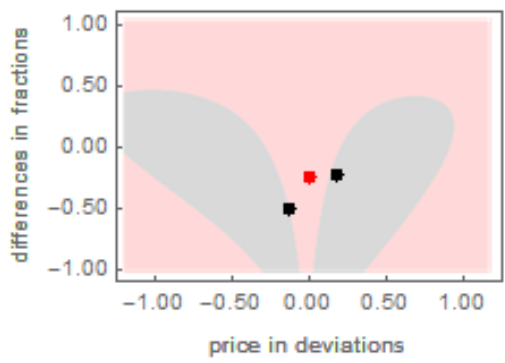

(b)

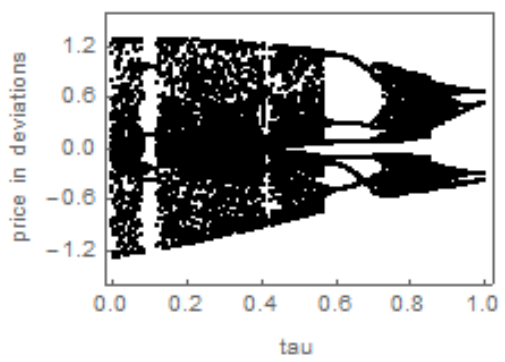

(d)

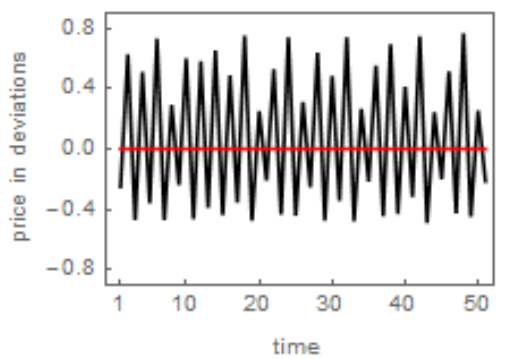

(f)

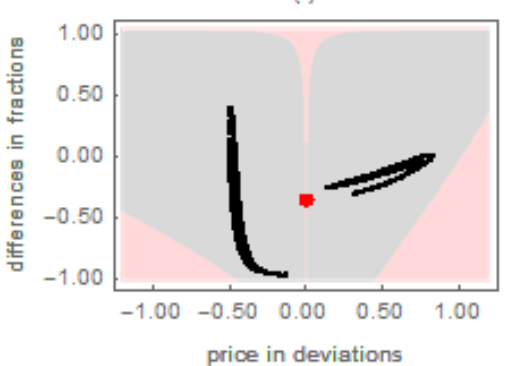

(h)

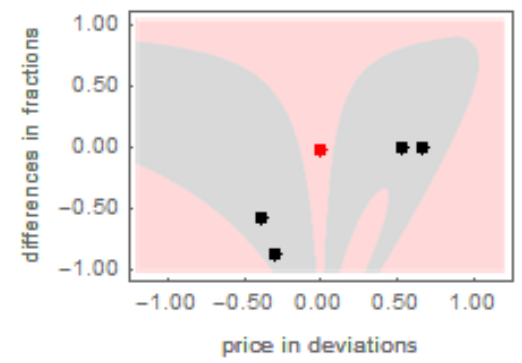

Figure 3: Coexisting attractors for case $X(L)$. (a) Bifurcation diagram for the intensity of choice with $\tau=0.5$. (b) Bifurcation diagram for the profit tax rate with $\beta=5$. (c) Time series for initial conditions $\left(\tilde{p}_{1}=0.1\right.$, $m_{1}=m^{*}$ ), red line, and $\left(\tilde{p}_{1}=0.25, m_{1}=m^{*}\right.$ ), black line, with $\beta=1.2$ and $\tau=0.5$. (d) Time series for initial conditions ( $\tilde{p}_{1}=0.001, m_{1}=m^{*}$ ), red line, and $\left(\tilde{p}_{1}=0.25, m_{1}=m^{*}\right.$ ), black line, with $\beta=5$ and $\tau=0.85$. (e) Basins of attraction and coexisting attractors with $\beta=1.2$ and $\tau=0.5$. (f) Basins of attraction and coexisting attractors for $\beta=5$ and $\tau=0.85$. (g) Basins of attraction and coexisting attractors with $\beta=1.2$ and $\tau=0.58$. (h) Basins of attraction and coexisting attractors for $\beta=5$ and $\tau=0.99$. 
(a)

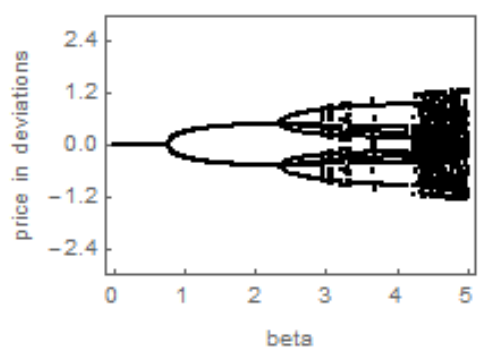

(c)

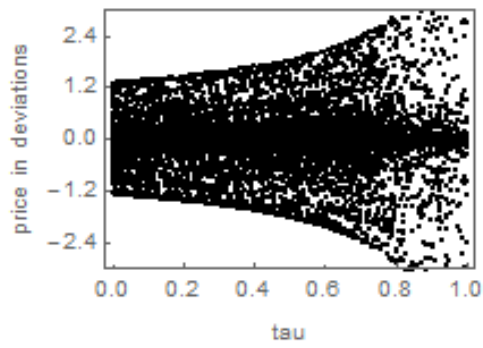

(e)

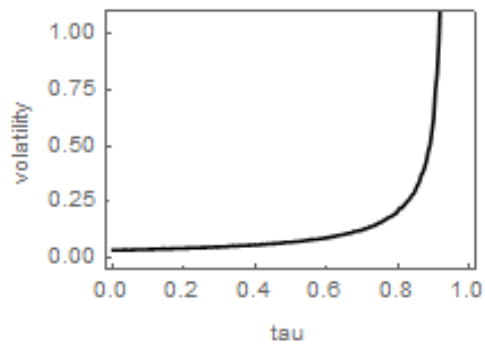

(g)

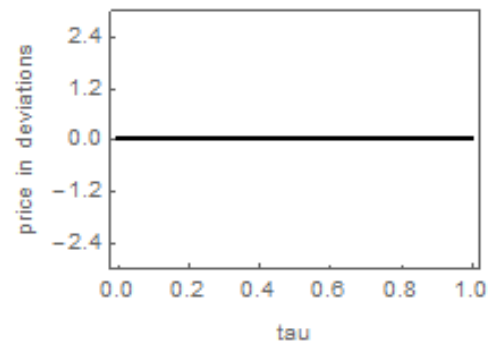

(i)

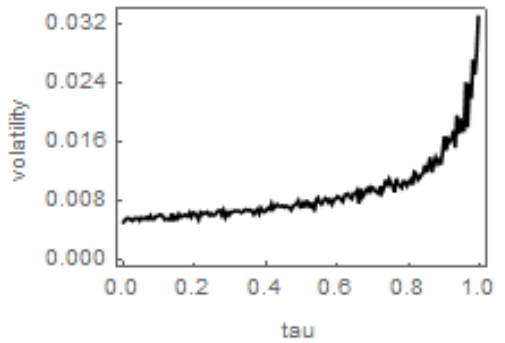

(b)

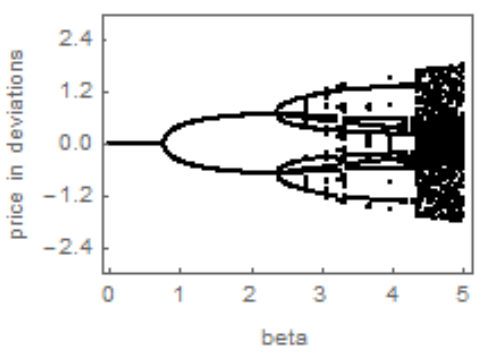

(d)

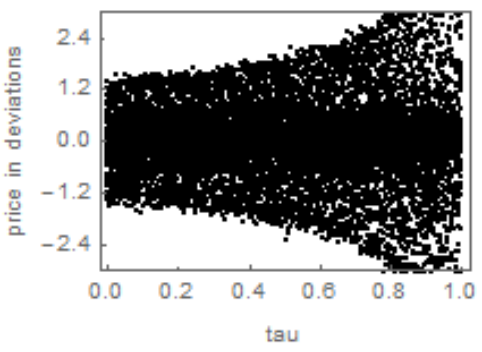

(f)

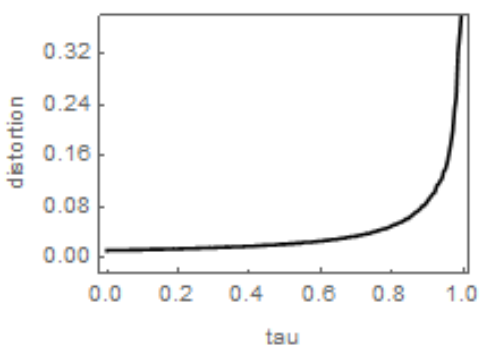

(h)

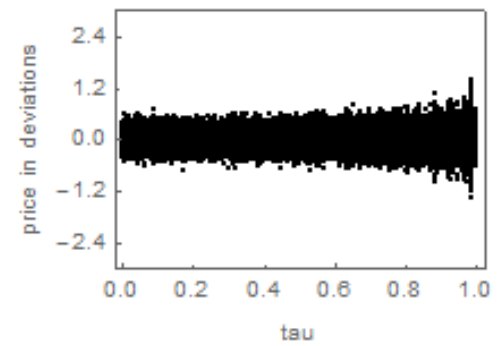

(j)

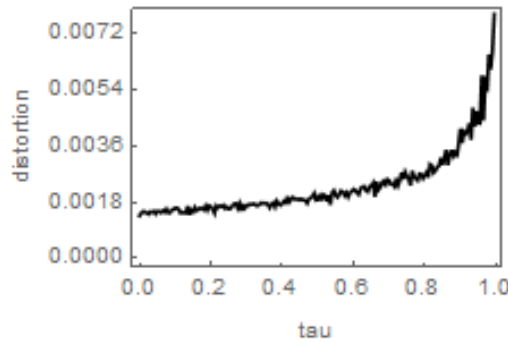

Figure 4: Dynamics for case $Y(H)$. (a) Bifurcation diagram for the intensity of choice with $\tau=0$. (b) Bifurcation diagram for the intensity of choice with $\tau=0.5$. (c) Bifurcation diagram for the profit tax rate with $\beta=5$. (d) Bifurcation diagram for the profit tax rate with $\beta=5$ and exogenous noise. (e) Volatility as a function of profit taxes with $\beta=5$ and exogenous noise. (f) Distortion as a function of profit taxes with $\beta=5$ and exogenous noise. (g) Bifurcation diagram for the profit tax rate with $\beta=0.75$. (h) Bifurcation diagram for the profit tax rate with $\beta=0.75$ and exogenous noise. (i) Volatility as a function of profit taxes with $\beta=0.75$ and exogenous noise. (j) Distortion as a function of profit taxes with $\beta=0.75$ and exogenous noise. 
(a)

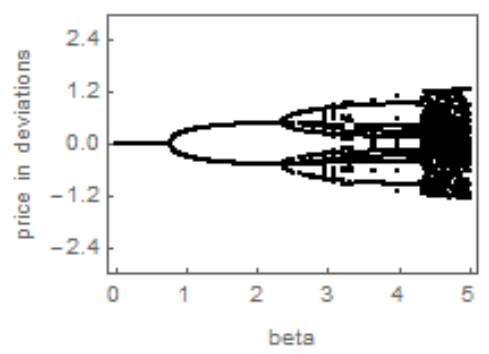

(c)

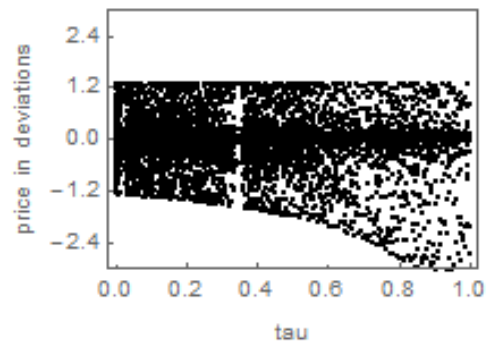

(e)

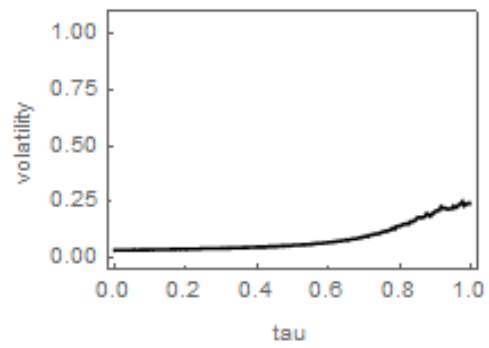

(g)

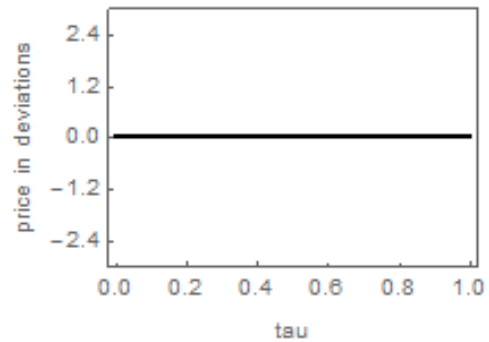

(i)

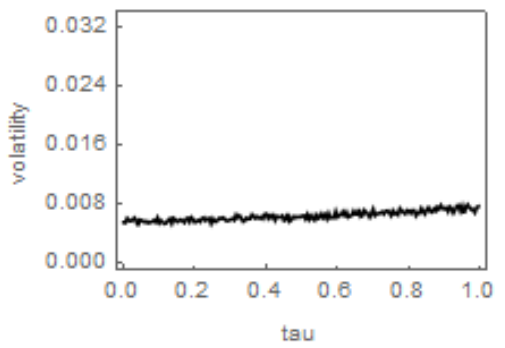

(b)

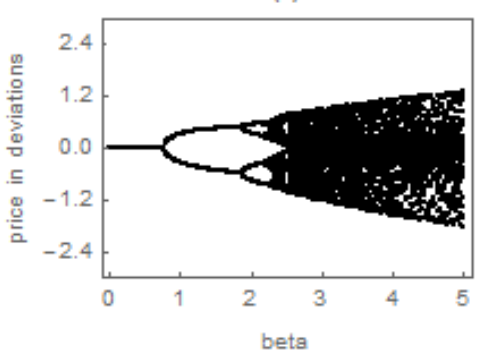

(d)

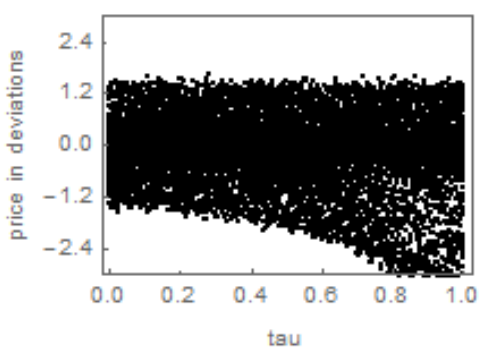

(f)

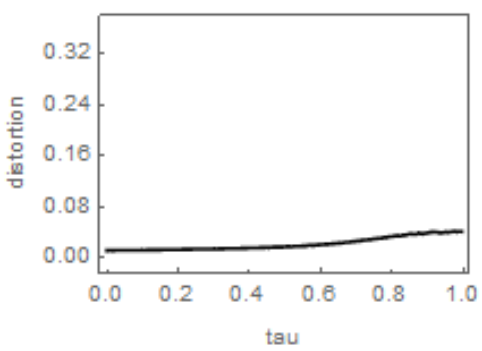

(h)

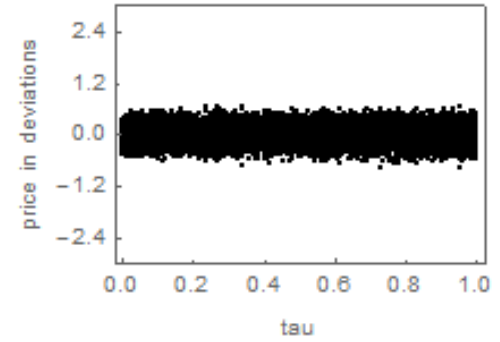

(j)

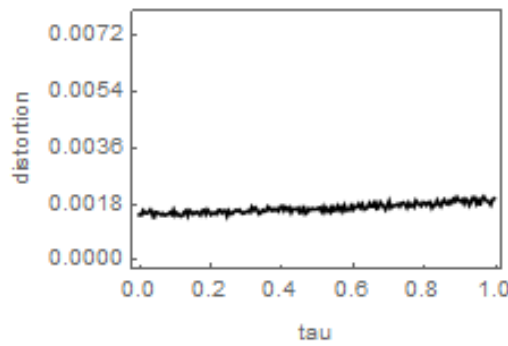

Figure 5: Dynamics for case $Y(L)$. (a) Bifurcation diagram for the intensity of choice with $\tau=0$. (b) Bifurcation diagram for the intensity of choice with $\tau=0.5$. (c) Bifurcation diagram for the profit tax rate with $\beta=5$. (d) Bifurcation diagram for the profit tax rate with $\beta=5$ and exogenous noise. (e) Volatility as a function of profit taxes with $\beta=5$ and exogenous noise. (f) Distortion as a function of profit taxes with $\beta=5$ and exogenous noise. (g) Bifurcation diagram for the profit tax rate with $\beta=0.75$. (h) Bifurcation diagram for the profit tax rate with $\beta=0.75$ and exogenous noise. (i) Volatility as a function of profit taxes with $\beta=0.75$ and exogenous noise. (j) Distortion as a function of profit taxes with $\beta=0.75$ and exogenous noise. 
(a)

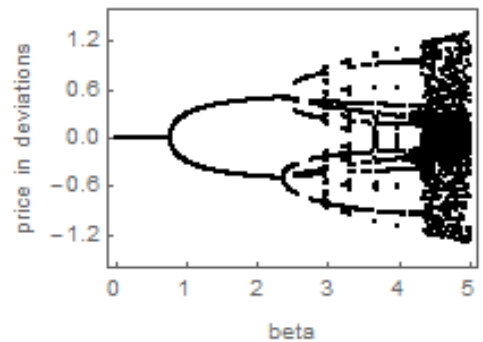

(c)

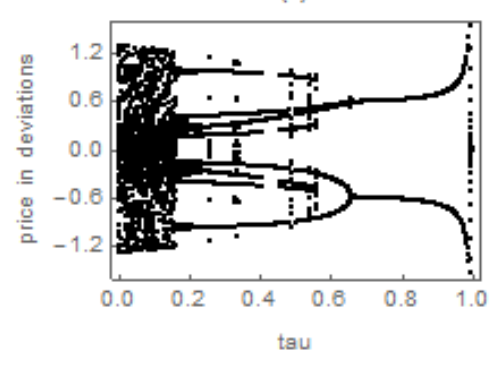

(e)

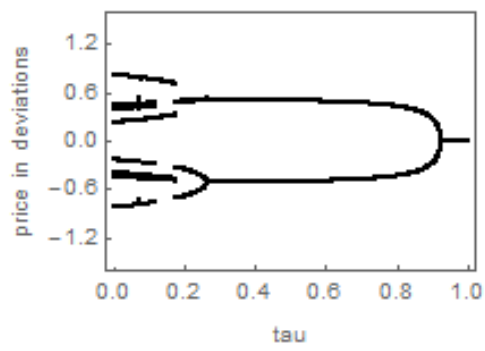

(g)

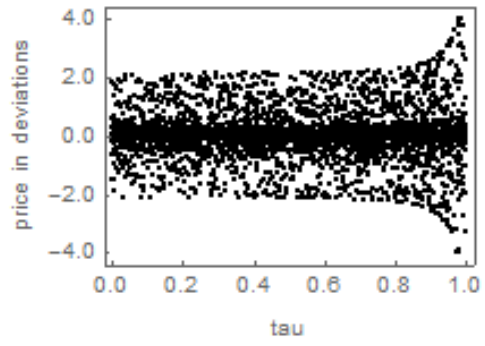

(b)

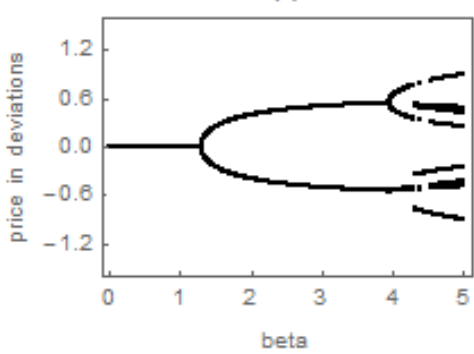

(d)

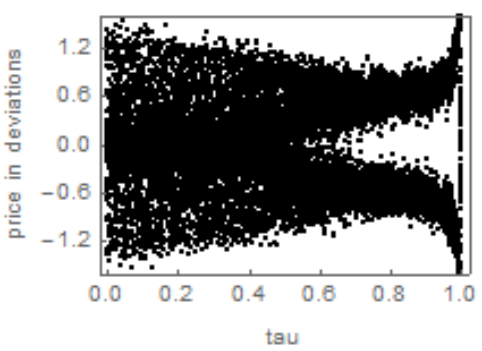

(f)

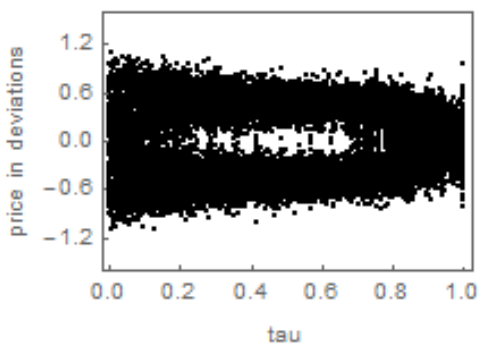

(h)

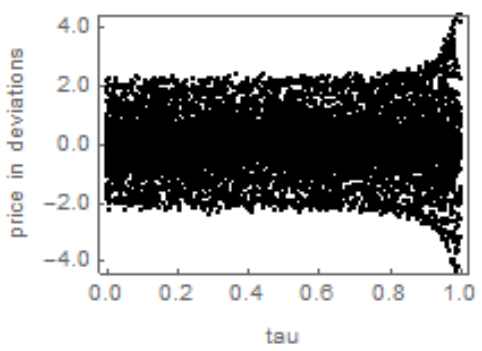

Figure 6: Dynamics for case $Z(H)$. (a) Bifurcation diagram for the intensity of choice with $\tau=0$. (b) Bifurcation diagram for the intensity of choice with $\tau=0.5$. (c) Bifurcation diagram for the profit tax rate with $\beta=5$. (d) Bifurcation diagram for the profit tax rate with $\beta=5$ and exogenous noise. (e) Bifurcation diagram for the profit tax rate with $\beta=3$. (f) Bifurcation diagram for the profit tax rate with $\beta=3$ and exogenous noise. (g) Bifurcation diagram for the profit tax rate with $\beta=25$. (h) Bifurcation diagram for the profit tax rate with $\beta=25$ and exogenous noise. 